\title{
ESTUDIO DE MÉTODOS QUÍMICOS DE REMOCIÓN DE CIANURO PRESENTE EN RESIDUOS DE CIANURACIÓN PROVENIENTES DEL PROCESO DE EXTRACCIÓN DE ORO DE VETA EN EL DEPARTAMENTO DE NARIÑO
}

\author{
JONNY ARIEL FAJARDOb, DIANA CAROLINA BURBANOb, EDITH JACKELINE \\ BURBANOb, NÉSTOR JAVIER APRAEZc Y MILTON ROSERO MOREANOa \\ milton.rosero@ucaldas.edu.co
}

Manizales, 2009-11-05 (Rev. 2010-03-28)

\section{RESUMEN}

Esta investigación se centró en la neutralización a nivel de laboratorio de residuos cianurados provenientes de la mina Nueva Esparta ubicada en el municipio de los Andes-Sotomayor (Nariño), con tres neutralizantes de tipo comercial en distintas relaciones en peso (gramos de neutralizante/gramos de $\mathrm{CN}$ - libre/total a neutralizar): peróxido de hidrógeno H2O2 2/1, 5/1 y 8/1, hipoclorito de sodio $\mathrm{NaOCl} 7 / 1,12 / 1$ y 17/1 y sulfato ferroso FeSO4 6/1, 12/1 y 18/1. Las arenas residuales se trataron así: lavado con agua, neutralización del agua de lavado y recirculación de la misma para un nuevo lavado; esto con el objeto de minimizar la cantidad de agua empleada. Después del análisis estadístico y teniendo en cuenta consideraciones técnicas, económicas y ambientales, se encontró que los mejores tratamientos para la remoción de cianuro libre y total son respectivamente las relaciones $2 / 1$ y 5/1 del $\mathrm{H} 2 \mathrm{O} 2$. Se realizó una caracterización fisicoquímica de la fuente de agua afectada por la actividad de la mina en estudio, de la solución pobre de cianuro y soluciones lixiviadas en laboratorio. En la evaluación de los metales $\mathrm{Fe}, \mathrm{Cu}, \mathrm{Ni}, \mathrm{Zn}, \mathrm{Mn}$ y $\mathrm{Pb}$ se observó que luego de la neutralización con H2O2, los metales analizados disminuyeron su concentración; con $\mathrm{NaOCl}$ se presentó un incremento en la mayoría, con una remoción mínima de plomo y manganeso. Con FeSO4, se obtuvo remoción para la mayoría, pero plomo y manganeso se incrementaron.

\section{PALABRAS CLAVE:}

Cianuro, remoción, cianuración, arenas residuales, minería del oro.

\section{STUDY OF CHEMICAL METHODS FOR CYANIDE REMOVAL FROM CYANIDATION WASTE COMING FROM THE GOLD VEIN EXTRACTION PROCESS IN THE DEPARTMENT OF NARIÑO}

\begin{abstract}
This research focused on laboratory-scale neutralization of cyanide wastes from the Nueva Esparta mine in the municipality of Los Andes, Sotomayor (Nariño) with three commercial type neutralizers in different weight ratios (neutralizer grams / CN-free/total to be neutralized): hydrogen peroxide $\mathrm{H} 2 \mathrm{O} 2$ 2/1, 5/1 and $8 / 1$, sodium hypochlorite $\mathrm{NaOCl} 7 / 1,12 / 1$ and $17 / 1$ and ferrous sulfate FeSO4 $6 / 1,12 / 1$ and 18/1. Residual sands were treated as follows: washing with water, neutralization of the washing water and circulation of the same water for a new washing process, this with the purpose of minimizing the quantity of water used. After the statistic analysis and taking into account technical, economical and environmental considerations, it was found that the best treatments for removing
\end{abstract}


free and total cyanide are respectively the relations $2 / 1$ and5/1 from $\mathrm{H} 2 \mathrm{O} 2$. A physical-chemical characterization of the water source affected by the activity on the poor cyanide solution and solutions lixiviated in the laboratory of the mine under study was performed. In the evaluation of the metals $\mathrm{Fe}, \mathrm{Cu}, \mathrm{Ni}, \mathrm{Zn}, \mathrm{Mn}$ and Pbit was observed that after the neutralization with con $\mathrm{H} 2 \mathrm{O} 2$, the analyzed metals diminished their concentration. There was an increase in the majority With $\mathrm{NaOCl}$ woth a minimum removal of lead and manganese. With FeSO4 there was removal for the majority but lead and manganese increased.

KEY WORDS: cyanide, removal, cyanide, residual sand, gold mining.

\section{INTRODUCCIÓN}

En las minas ubicadas en la Cordillera Occidental del departamento de Nariño se practica la técnica de cianuración para la recuperación del oro de veta, la cual se realiza de una manera inapropiada afectando tanto la salud de las personas como al medio ambiente (ver Figura 1). La principal causa del impacto sanitario y ambiental es el inadecuado manejo de los residuos de dicho proceso (arenas cianuradas) los cuales son depositados al aire libre sin tratamiento previo cerca de las fuentes de agua generando emisiones tóxicas a la atmósfera, al suelo y el agua. $^{1}$

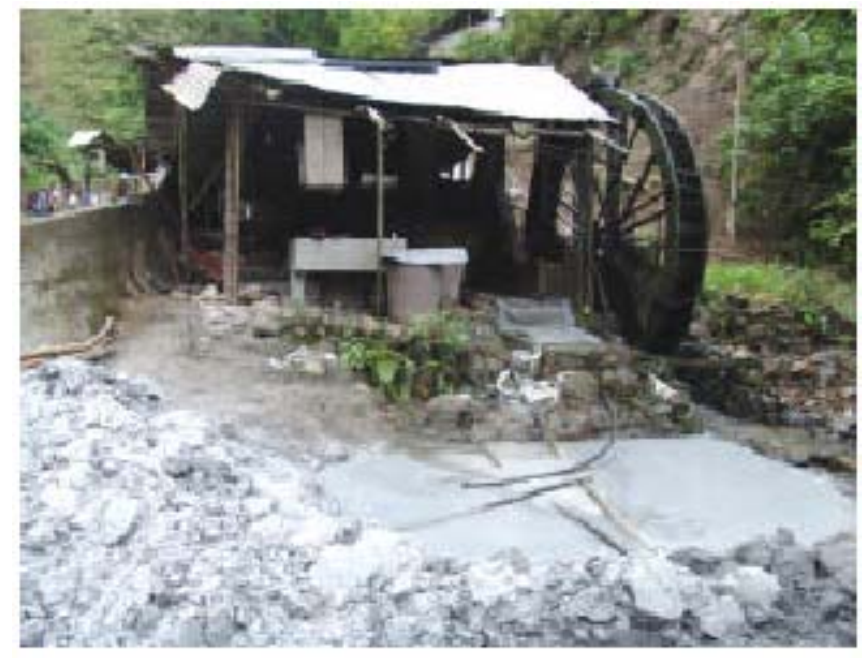

Figura 1. Disposición inadecuada de las arenas cianuradas en los procesos de beneficio de la minería del oro, Sotomayor (Nariño).

El cianuro es altamente tóxico, sin embargo puede ser sometido a una degradación química, natural o biológica para disminuir su poder contaminante. ${ }^{2}$ Dentro de los métodos químicos tenemos:

Precipitación con sulfato ferroso: este proceso es utilizado para transformar el cianuro libre a ferrocianuro y/o ferricianuro que son compuestos más estables considerados de baja toxicidad y se realiza a un pH óptimo de 7,5-10,5 y el hierro se adiciona como sulfato ferroso. ${ }^{3}$ Las reacciones del proceso son: 


$$
\begin{aligned}
& \mathrm{Fe}^{+2}+6 \mathrm{CN}^{-}+1 / 4 \mathrm{O}_{2}+\mathrm{H}^{+} \longrightarrow \mathrm{Fe}(\mathrm{CN})_{6}^{-3}+1 / 2 \mathrm{H}_{2} \mathrm{O} \\
& 4 \mathrm{Fe}^{+2}+3 \mathrm{Fe}(\mathrm{CN})_{6}^{-3}+1 / 4 \mathrm{O}_{2}+\mathrm{H}^{+} \longrightarrow \mathrm{Fe}_{4}\left[\mathrm{Fe}(\mathrm{CN})_{6}\right]_{3}+1 / 2 \mathrm{H}_{2} \mathrm{O}
\end{aligned}
$$

Oxidación con peróxido de hidrógeno: oxidación catalizada con $\mathrm{Cu}+2$, el cual se adiciona como sulfato de cobre en medio alcalino, formándose un complejo con el ion cianuro (tetracianocuprato) el cual tiene mayor afinidad con el peróxido. Éste es un proceso nuevo, simple, con mínimos requisitos de supervisión y bajos costos; el peróxido de hidrógeno es un compuesto no tóxico y altamente oxidante que puede romper fácilmente el compuesto de cianuro. ${ }^{4}$

$$
\begin{aligned}
& 3\left[\mathrm{Cu}(\mathrm{CN})_{4}\right]^{-3}+6 \mathrm{H}_{2} \mathrm{O}_{2} \longrightarrow 3 \mathrm{Cu}^{+2}+12 \mathrm{OCN}^{-}+12 \mathrm{H}^{+} \\
& \mathrm{OCN}^{-}+2 \mathrm{H}_{2} \mathrm{O} \rightarrow \mathrm{CO}_{3}=+\mathrm{NH}_{4}^{+}
\end{aligned}
$$

Tratamiento con hipoclorito de calcio: consiste en la oxidación de cianuro a cianato de calcio y luego a pH ácido, éste se descompone en amoniaco y bicarbonato; el uso de estos productos tiene desventajas como la formación de compuestos tóxicos como cloruro de cianógeno y organoclorados; se libera al medio ambiente cloro residual y cloruros, requiere condiciones alcalinas fuertes y el consumo del oxidante puede ser muy alto. ${ }^{5}$

$$
\begin{aligned}
& 2 \mathrm{NaCN}+\mathrm{Ca}(\mathrm{ClO})_{2}+4 \mathrm{H}_{2} \mathrm{O} \longrightarrow 2 \mathrm{CNCl}+\mathrm{Ca}(\mathrm{OH})_{2} \\
& \mathrm{Ca}(\mathrm{CNO})_{2}+4 \mathrm{H}_{2} \mathrm{O} \rightarrow\left(\mathrm{NH}_{4}\right)_{2} \mathrm{CO}_{3}+\mathrm{CaCO}_{3} \\
& 2 \mathrm{CNCl}+\mathrm{Ca}(\mathrm{OH})_{2} \rightarrow \mathrm{Ca}(\mathrm{CNO})_{2}+2 \mathrm{H}_{2} \mathrm{O}+\mathrm{CaCl}_{2}
\end{aligned}
$$

Con este estudio se pretende evaluar a nivel de laboratorio tres diferentes tipos de tratamientos químicos: oxidación con $\mathrm{H} 2 \mathrm{O} 2$, oxidación con $\mathrm{NaOCl}$ y precipitación con $\mathrm{FeSO} 4$ para la remoción de cianuro y metales pesados como $\mathrm{Fe}, \mathrm{Zn}, \mathrm{Ni}, \mathrm{Mn}, \mathrm{Pb}$ y $\mathrm{Cu}$, presentes en las arenas residuales provenientes del proceso de cianuración por percolación en el proceso de beneficio de oro, en la mina Nueva Esparta (Nariño) estableciendo las condiciones óptimas para conseguir, una vez escalados los hallazgos de esta investigación, una reducción importante de las emisiones tóxicas de estos compuestos al ambiente.

\section{MATERIALES Y MÉTODOS}

\section{Muestreo}

Se hizo el día de la descarga de las arenas cianuradas del tanque de cianuración, tomando tres muestras compuestas de la parte superior, media y fondo del tanque.

\section{Caracterización fisicoquímica de la muestra}

Se determinó pH (mediante pH-metro), metales como hierro, cobre, zinc, níquel, manganeso y plomo (por absorción atómica con llama) previa digestión ácida'; 
sulfatos (por el método turbidimétrico), cianuro total (mediante destilación) y cianuro libre (por titulación con AgNO3 y por test con reactivos) a las muestras de arenas cianuradas, aguas de lavados de arenas y lixiviados de arenas a 24 horas; al igual que a muestras de la quebrada aledaña a los molinos de la mina y soluciones pobres de los tanques de cianuración. Análisis todos realizados en los laboratorios especializados de la Universidad de Nariño según el Standard Methods de la AWWA APHA 19a edición.

\section{Ensayos de tratabilidad a escala laboratorio}

a) La lixiviación se realizó tomando $10 \mathrm{~g}$ de arena contenidos en un tejido con tamaño de poro de $50 \mu \mathrm{m}$, sometidos a agitación constante por 24 horas en un litro de agua destilada. El método espectrofotométrico para cianuro por test con reactivos (Merck Spectroquant) fue validado según las guías de la CEM/OPS del Centro Panamericano de Ingeniería Sanitaria CEPIS.

b) Neutralización de cianuro: se aplicó a las arenas residuales del proceso de cianuración, que se trataron por el siguiente método: lavado con agua, neutralización del agua de lavado y recirculación de la misma para un nuevo lavado. Esto con el objeto de minimizar la cantidad de agua empleada en los lavados. El contenido de cianuro se midió después de cada ciclo, realizando cuatro en total. Para realizar este procedimiento se construyeron unidades experimentales que consisten en columnas de pvc de 2 pulgadas de diámetro y $50 \mathrm{~cm}$ de longitud, con un filtro en la parte inferior y un desagüe para recoger las aguas de lavado (ver Figura 2). En cada columna se trató 1 kilogramo de arena residual.

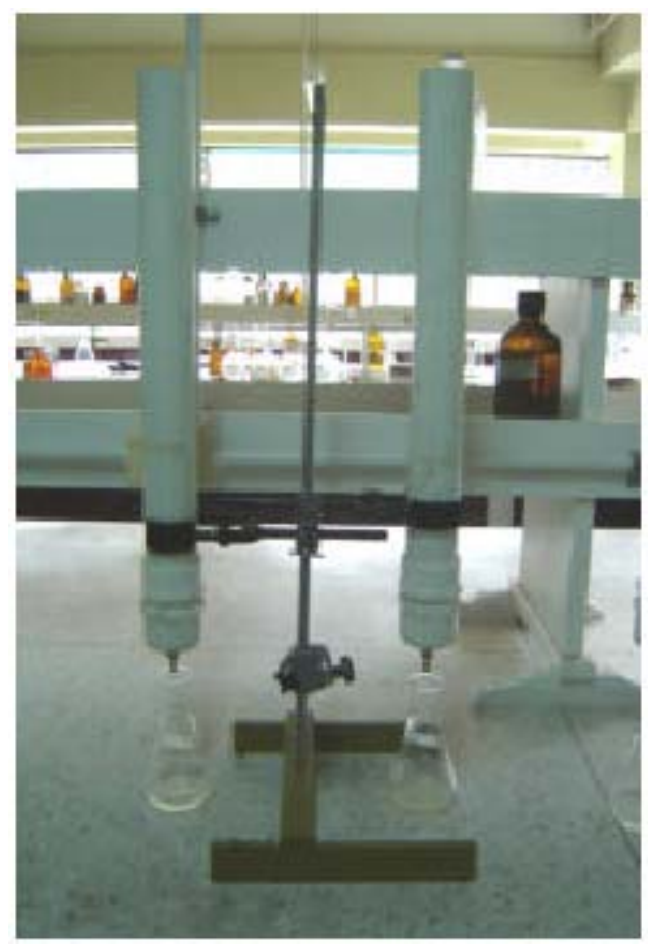

Figura 2. Unidad experimental ensayos de tratabilidad para arenas cianuradas mediante neutralización de cianuro con diferentes agentes químicos. 
Para cada uno de los neutralizantes químicos comerciales se estudiaron tres diferentes relaciones en peso. Peróxido de hidrógeno: 2/1, 5/1 y 8/1 g H2O2/g $\mathrm{CN}$ - con un tiempo de reacción de 3 horas y $\mathrm{pH}$ de 9-11,5; hipoclorito de sodio: $7 / 1,12 / 1$ y $17 / 1 \mathrm{~g} \mathrm{HClO} / \mathrm{g} \mathrm{CN}$ - con un tiempo de reacción de 2 horas y $\mathrm{pH}$ mayor a 11; y sulfato ferroso: 6/1, 12/1 y 18/1 g FeSO4/g CN- con un tiempo de reacción de 1 hora y un pH de 7,5 a 10,5. Cada relación se consideró como un tratamiento para un total de nueve, que se analizaron estadísticamente con base en un diseño irrestrictamente al azar (DIA).

\section{RESULTADOS Y DISCUSIÓN}

En la Tabla 1 se presentan los datos de los parámetros fisicoquímicos evaluados en cuatro tipos de muestras distintas, tanto de muestras de agua de influencia directa del proceso de extracción como de los ensayos de tratabilidad realizados a nivel de laboratorio.

Tabla 1. Caracterización fisicoquímica muestras de agua y ensayos de tratabilidad.

\begin{tabular}{|c||c||c||c|c||c||c||c|c||c|}
\hline \hline Muestra & $\mathrm{pH}$ & $\begin{array}{c}\mathrm{SO}_{4}= \\
(\mathrm{ppm})\end{array}$ & $\begin{array}{c}\mathrm{Fe} \\
(\mathrm{ppm})\end{array}$ & $\begin{array}{c}\mathrm{Cu} \\
(\mathrm{ppm})\end{array}$ & $\begin{array}{c}\mathrm{Zn} \\
(\mathrm{ppm})\end{array}$ & $\begin{array}{c}\mathrm{Pb} \\
(\mathrm{ppm})\end{array}$ & $\begin{array}{c}\mathrm{Mn} \\
\mathrm{ppm})\end{array}$ & $\begin{array}{c}\mathrm{Ni} \\
(\mathrm{ppm})\end{array}$ & $\begin{array}{c}\mathrm{CN}^{-} \\
\text {Libre } \\
(\mathrm{ppm})\end{array}$ \\
\hline 1 & 6,15 & 7,76 & 0,114 & $2 \times 10^{-3}$ & $\begin{array}{c}6 \times 10 \\
4\end{array}$ & 0,024 & $2 \times 10^{-3}$ & 0,094 & $<4 \times 10^{-3}$ \\
\hline 2 & 6,85 & 10,1 & 0,13 & 0,01 & 0,112 & 0,04 & 0,01 & 0,102 & $<4 \times 10^{-3}$ \\
\hline 3 & 9,87 & 308,0 & 12,0 & 190,5 & 910,0 & 0,24 & 0,01 & 3,84 & 433,33 \\
\hline 4 & $10^{*}$ & 515,5 & 0,64 & 0,32 & 0,54 & 0,65 & 0,01 & 0,57 & 0,061 \\
\hline
\end{tabular}

Muestra 1: Agua quebrada Honda, $50 \mathrm{~m}$ antes de molinos.

Muestra 2: Agua quebrada Honda, $50 \mathrm{~m}$ después de molinos.

Muestra 3: Solución pobre de cianuro del sistema de cianuración.

Muestra 4: Lixiviación $10 \mathrm{~g}$ de arena

Ninguno de los parámetros fisicoquímicos analizados a las muestras de agua antes y después de los molinos de la mina, están por encima de los límites permisibles según el Decreto 1594 de 1984, sin embargo se observa un ligero incremento en la concentración de dichos parámetros $50 \mathrm{~m}$ después de los molinos muy probablemente por la actividad de esta mina.

La Muestra 4 simula la lixiviación natural de las arenas cuando son depositadas a orillas de las quebradas sin un previo tratamiento. No se encontró una correlación directa entre un mayor contenido de metal en el mineral y una mayor lixiviación de dichos metales, debido a la diferencia de solubilidad de los minerales presentes en el material de la mina.

En la solución pobre de cianuro se encontró que las concentraciones de níquel y cobre están por encima de los límites permisibles para el vertimiento de residuos líquidos (Decreto 1594), esto se debe a la composición mineralógica del material. Los niveles de zinc, hierro, manganeso y sulfatos (no contemplados en el 
Decreto) se encuentran en concentraciones relativamente altas, debido a la presencia de minerales como pirita (FeS2), blenda (ZnS), esfarelita (ZnFe)S y otros sulfuros que por acción del oxígeno se oxidan a sulfatos, ocasionando una liberación de metales. El alto nivel de zinc también se debe a la utilización de viruta de zinc para precipitar el oro. El contenido de cianuro presente en esta solución es bastante elevado, lo cual representa un riesgo inminente para el medio ambiente si fueran descargadas sin un previo tratamiento.

Tabla 2. Caracterización de arena antes de neutralizar.

\begin{tabular}{|c|c||}
\hline $\begin{array}{c}\text { Parámetros } \\
\text { determinados }\end{array}$ & $\begin{array}{c}\text { Concentración en } \\
\mathrm{mg} / \mathrm{kg} \text { arena }\end{array}$ \\
\hline Hierro & 154000 \\
\hline Cobre & 130 \\
\hline Zinc & 900 \\
\hline Plomo & 650 \\
\hline Manganeso & 780 \\
\hline Níquel & 124 \\
\hline Sulfatos & 5026 \\
\hline Cianuro total & 436,8 \\
\hline \hline
\end{tabular}

Según la Tabla 2, las altas concentraciones de hierro y sulfatos se deben a la alta cantidad de pirita presente en el mineral de veta, los cuales sufren un proceso de oxidación liberando sulfatos y cationes como el hierro, produciendo acidez y turbidez en las aguas.

Los sulfuros se descomponen de acuerdo con la siguiente reacción ${ }^{1}$ :

$$
\mathrm{FeS}_{2}+7 / 2 \mathrm{O}_{2}+\mathrm{H}_{2} \mathrm{O} \longrightarrow \mathrm{Fe}^{2+}+2 \mathrm{SO}_{4}{ }^{2-}+2 \mathrm{H}^{+}
$$

Tabla 3. Caracterización de soluciones de lavado inicial (antes de neutralización).

\begin{tabular}{||c||c||c||}
\hline $\begin{array}{c}\text { Parámetros } \\
\text { determinados }\end{array}$ & $\begin{array}{c}\text { Concentración en } \\
\mathrm{mg} / \text { L solución* }\end{array}$ & $\begin{array}{c}\text { Cantidad en } \\
\mathrm{mg} * *\end{array}$ \\
\hline Hierro & 14,15 & 1,981 \\
\hline Cobre & 220,5 & 30,87 \\
\hline Zinc & 60 & 8,4 \\
\hline Plomo & 0,245 & 0,0343 \\
\hline Manganeso & 0,055 & 0,0077 \\
\hline Níquel & 2,7 & 0,378 \\
\hline Sulfatos & 4600 & 592,5 \\
\hline
\end{tabular}


* Concentración de solución del lavado inicial de 1 kg de arena (volumen de la solución recogida después de lavar: $140 \mathrm{ml}$ ).

** Cantidad extraída del lavado inicial de 1 kg de arena.

En la Tabla 3, se observa que al comparar la cantidad extraída de $1 \mathrm{~kg}$ de arena con la concentración en $\mathrm{mg} / \mathrm{kg}$ para los parámetros analizados se aprecia que sólo una pequeña cantidad de dichas sustancias es solubilizada.

El método espectrofotométrico de test con reactivos se encontró que es repetible, reproducible y exacto. Los límites de detección y cuantificación son: 0,004 ppm de $\mathrm{CN}$ - y 0,014 ppm de $\mathrm{CN}-$, respectivamente.

Tabla 4. Pruebas de comparación múltiple para los diferentes tratamientos de neutralización para cianuro libre.

\begin{tabular}{|c|c|c|c|}
\hline Tratamientos & Medias & $\begin{array}{l}\text { Grupos Homogéneos } \\
\text { Prueba de Tukey }\end{array}$ & $\begin{array}{l}\text { Grupos Homogéneos } \\
\text { Prueba de Duncan }\end{array}$ \\
\hline $\mathrm{T} 9=\mathrm{FeSO}_{4} 18 / 1$ & 97,955 & A & A \\
\hline $\mathrm{T} 2=\mathrm{H}_{2} \mathrm{O}_{2} 5 / 1$ & 97,585 & $\mathrm{AB}$ & $\mathrm{AB}$ \\
\hline $\mathrm{T} 1=\mathrm{H}_{2} \mathrm{O}_{2} 2 / 1$ & 97,510 & $\mathrm{AB}$ & $\mathrm{AB}$ \\
\hline $\mathrm{T} 6=\mathrm{NaClO} 17 / 1$ & 97,310 & $\mathrm{ABC}$ & $\mathrm{AB}$ \\
\hline $\mathrm{T} 3=\mathrm{H}_{2} \mathrm{O}_{2} \mathrm{~S} / 1$ & 97,180 & $\mathrm{ABC}$ & $\mathrm{ABC}$ \\
\hline $\mathrm{T} 8=\mathrm{FeSO}_{4} 12 / 1$ & 96,935 & $\mathrm{ABC}$ & $\mathrm{BC}$ \\
\hline $\mathrm{T} 7=\mathrm{FeSO}_{4} 6 / 1$ & 96,295 & $\mathrm{~B} \mathrm{C}$ & $\mathrm{CD}$ \\
\hline $\mathrm{T} 5=\mathrm{NaClO} 12 / 1$ & 95,945 & $\mathrm{C}$ & $\mathrm{D}$ \\
\hline $\mathrm{T} 4=\mathrm{NaClO} 7 / 1$ & 93,085 & $\mathrm{D}$ & $\mathrm{E}$ \\
\hline
\end{tabular}

Después de realizado el análisis de varianza (ver Tabla 4), que determinó que existen diferencias significativas entre los tratamientos, se efectuaron las pruebas de Tukey y Duncan para los porcentajes de remoción de cianuro libre. Según estas pruebas se encontraron varios grupos homogéneos diferentes entre sí, de los cuales se recomienda el Grupo A por presentar los más altos porcentajes de remoción. Sin embargo, desde el punto de vista técnico, económico y ambiental se recomienda el tratamiento $\mathrm{H} 2 \mathrm{O} 2$ 2/1 por presentar ventajas con respecto a los otros neutralizantes como: la no formación de excesiva cantidad de precipitado a diferencia de sulfato ferroso, la no generación de cloro residual a diferencia del hipoclorito de sodio, lo cual puede generar problemas cuando se tratan grandes cantidades de arenas puesto que se incrementa la cantidad de cloro que es precursor de organoclorados, sustancias tóxicas de difícil degradación; además el exceso de peróxido se puede descomponer en agua y oxígeno.

Tabla 5. Pruebas de comparación múltiple para los diferentes tratamientos de neutralización para cianuro total. 


\begin{tabular}{||c||c||c||}
\hline Tratamientos & Medias & $\begin{array}{c}\text { Grupos Homogéneos } \\
\text { Prueba de Duncan }\end{array}$ \\
\hline $\mathrm{T} 6=\mathrm{NaClO} 17 / 1$ & 82,140 & $\mathrm{~A}$ \\
\hline $\mathrm{T} 3=\mathrm{H}_{2} \mathrm{O}_{2} 8 / 1$ & 80,950 & $\mathrm{~A}$ \\
\hline $\mathrm{T} 4=\mathrm{NaClO} 7 / 1$ & 80,950 & $\mathrm{~A}$ \\
\hline $\mathrm{T} 8=\mathrm{FeSO}_{4} 12 / 1$ & 80,950 & $\mathrm{~A}$ \\
\hline $\mathrm{T} 5=\mathrm{NaClO}_{12} / 1$ & 79,760 & $\mathrm{~A}$ \\
\hline $\mathrm{T} 9=\mathrm{FeSO}_{4} 18 / 1$ & 77,380 & $\mathrm{~A} \mathrm{~B}$ \\
\hline $\mathrm{T} 2=\mathrm{H}_{2} \mathrm{O}_{2} 5 / 1$ & 73,805 & $\mathrm{~A} \mathrm{~B}$ \\
\hline $\mathrm{T} 1=\mathrm{H}_{2} \mathrm{O}_{2} 2 / 1$ & 69,050 & $\mathrm{~B}$ \\
\hline $\mathrm{T} 7=\mathrm{FeSO}_{4} 6 / 1$ & 69,030 & $\mathrm{~B}$ \\
\hline
\end{tabular}

Teniendo en cuenta la prueba de Duncan (ver Tabla 5), se encontraron dos grupos de tratamientos de los cuales el A es mejor puesto que se obtienen los más altos porcentajes de remoción de cianuro total; sin embargo considerando las ventajas técnicas, económicas y ambientales mencionadas para el peróxido de hidrógeno, se recomienda utilizar este neutralizante en su relación 5/1.

Después de la neutralización de las arenas con peróxido de hidrógeno se presenta una considerable disminución de cobre, zinc y manganeso, los cuales son arrastrados en cada ciclo de lavado quedando en solución; esto debido a que este neutralizante es un oxidante fuerte de rápida reacción en medios básicos; en el caso del plomo y níquel se logró una menor remoción alrededor del 56\% y $31 \%$, respectivamente. Con el hipoclorito de sodio se presentó un incremento de la concentración de los metales cobre, zinc, níquel, manganeso y plomo; a excepción del manganeso y plomo con la relación 7/1 donde la remoción es mínima. Con el sulfato ferroso se obtuvo remoción considerable para el zinc y níquel con porcentajes algo superiores al 70\%. La remoción de cobre y manganeso fue mínima y para la relación 18/1 la concentración de manganeso se incrementó. En el caso del plomo también se presentó un incremento.

\section{CONCLUSIONES}

- Existen fuertes indicios de que el agua de la quebrada Honda se ve afectada por la actividad de la mina Nueva Esparta, lo cual se corrobora por el incremento en la concentración de los parámetros analizados en el agua después de las instalaciones de la mina.

- En la solución pobre de cianuro de la mina Nueva Esparta, las concentraciones de níquel y cobre están por encima de los límites permisibles para el vertimiento de residuos líquidos de acuerdo al Decreto 1594.

- El mejor tratamiento para la remoción de cianuro libre es el H2O2 en la relación $2 / 1$, porque se obtienen los más altos porcentajes de remoción, además presenta ventajas sobre los otros tratamientos, desde el punto de vista técnico, económico y ambiental.

- Estadísticamente se comprobó que los mejores porcentajes de remoción de cianuro total se obtuvieron con el hipoclorito de sodio en todas sus relaciones, las mayores relaciones del peróxido de hidrógeno y sulfato 
ferroso; sin embargo, se sugiere la aplicación del peróxido de hidrógeno en su relación 5/1 por las consideraciones antes mencionadas.

- En soluciones de lavado de arenas se presenta un elevado porcentaje de remoción de $\mathrm{Fe}, \mathrm{Cu}$ y $\mathrm{Zn}$ para todos los neutralizantes. El Mn y Pb sólo son removidos por $\mathrm{H} 2 \mathrm{O} 2$; mientras que con $\mathrm{NaClO}$ y $\mathrm{FeSO} 4$ se presenta un ligero aumento en su concentración.

- En arenas se aprecia un gran porcentaje de remoción de Fe con todos los neutralizantes. El H2O2 logró remoción para los metales restantes, mientras que con $\mathrm{NaClO}$ se presentó aumento de concentración para los mismos.

- En soluciones lixiviadas se dio remoción para $\mathrm{Fe}, \mathrm{Cu}, \mathrm{Zn}, \mathrm{Pb}$ y $\mathrm{Ni}$ con porcentajes disímiles. Para el Mn no se presentó remoción con H2O2; y con $\mathrm{NaClO}$ y FeSO4 se obtuvo un ligero incremento.

\section{REFERENCIAS}

1. Delgado, D. y Rodríguez, V. (2003). Caracterización minero ambiental del proceso de cianuración utilizado en el proceso de extracción de oro en el departamento de Nariño. Trabajo de grado para optar al título de Ingeniero Sanitario y Ambiental. Universidad Mariana, Facultad de Ingeniería Sanitaria y Ambiental, Pasto. 18p.

2. Wotruba, H., Hruschka, F.; Hentschel, T.; Priestel, M. (1998). Manejo ambiental en la pequeña minería. MEDMIN-COSUDE, La Paz, Bolivia. p. 155.

3. Mudder, T. How to treat cyanide. [en línea]. Obtenido el 11-11-2004, desde: http://www.cyantist.comltreatment.htm

4. Solvay. Cyanide detoxification with hidrogen peroxide. [en línea]. Obtenido el 09-10-2001, desde: http://www.solvayinterox.com

5. Smith, A. y Mudder, T. (1991). The chemistry and treatment of cyanidation wastes. Londres, Reino Unido: Mining Journal Books. 235p.

6. Sierra, J. (1988). Análisis de aguas y aguas residuales. Medellín: Universidad de Antioquia, Facultad de Ingeniería. p. 55-60.

a. Universidad de Caldas, Departamento de Química. Manizales, Colombia.

b. Universidad de Nariño, Departamento de Química. San Juan de Pasto, Colombia.

c. Empresa Metropolitana de Aseo EMAS S.A. E.S.P. San Juan de Pasto, Colombia. 